\title{
PARAMETRIZED MEASURING AND CLUB GUESSING
}

\author{
DAVID ASPERÓ AND JOHN KRUEGER
}

\begin{abstract}
We introduce Strong Measuring, a maximal strengthening of J. T. Moore's Measuring principle, which asserts that every collection of fewer than continuum many closed bounded subsets of $\omega_{1}$ is measured by some club subset of $\omega_{1}$. The consistency of Strong Measuring with the negation of $\mathrm{CH}$ is shown, solving an open problem from 2 about parametrized measuring principles. Specifically, we prove that Strong Measuring follows from MRP together with Martin's Axiom for $\sigma$-centered forcings, as well as from BPFA. We also consider strong versions of Measuring in the absence of the Axiom of Choice.
\end{abstract}

Club guessing principles at $\omega_{1}$ are well-studied natural weakenings of Jensen's $\diamond$ principle. Presented in a general form, they assert the existence of a sequence $\vec{C}=\left\langle c_{\alpha}: \alpha \in \omega_{1} \cap \operatorname{Lim}\right\rangle$, where each $c_{\alpha}$ is a club of $\alpha$, such that $\vec{C}$ guesses clubs of $\omega_{1}$ in some suitable sense. $\vec{C}$ guessing a club $D$ of $\omega_{1}$ usually means that there is some (equivalently, stationarily many) $\delta \in D$ such that $c_{\delta} \cap D$ is a suitably large subset of $c_{\delta}$; for example, we could require that $c_{\delta} \subseteq D$, in which case the resulting statement is called club guessing, or that $c_{\delta} \cap D$ is cofinal in $\delta$, in which case we call the resulting statement very weak club guessing.

Unlike the case of their versions at cardinals higher than $\omega_{1}$, for which there are non-trivial positive ZFC theorems (see, for example, [14]), club guessing principles at $\omega_{1}$ are independent of ZFC. On the one hand, all of these principles obviously follow from $\diamond$, and hence they hold in $L$, and they can always be forced by countably closed forcing. On the other hand, classical forcing axioms at the level of $\omega_{1}$, such as the Proper Forcing Axiom (PFA), imply the failure of even the weakest of these principles. It should nevertheless be noted that Martin's Axiom $+\neg \mathrm{CH}$ is compatible with Club Guessing. This is because Martin's Axiom can always be forced by a c.c.c. forcing, and the fact that every club of $\omega_{1}$ in a generic extension via a c.c.c. forcing contains a club of $\omega_{1}$ from the ground model implies that a club-guessing sequence from the ground model remains club-guessing in the extension. (On the other hand, this is of course not the case for $\diamond$ since the negation of $\mathrm{CH}$ violates $\diamond$.

Measuring is a particularly strong failure of Club Guessing due to J. T. Moore ([8]). Let $X$ and $Y$ be countable subsets of $\omega_{1}$ with the same supremum $\delta$. We say that $X$ measures $Y$ if there exists $\beta<\delta$ such that $X \backslash \beta$ is either contained in, or disjoint from, $Y$. Measuring is the statement that for any sequence $\left\langle c_{\alpha}: \alpha \in\right.$

Date: August 2018; revised July 2019.

The first author acknowledges support of EPSRC Grant EP/N032160/1. The second author was partially supported by the National Science Foundation Grant No. DMS-1464859.

2010 Mathematics Subject Classification: Primary 03E05, 03E35; Secondary 03E57.

Key words and phrases. Strong Measuring, Club Guessing, MRP, BPFA. 
$\left.\omega_{1} \cap \operatorname{Lim}\right\rangle$, where each $c_{\alpha}$ is a closed subset of $\alpha$, there exists a club $D \subseteq \omega_{1}$ such that for all limit points $\delta \in D$ of $D, D \cap \delta$ measures $c_{\delta}$.

Measuring can be viewed as a strong negation of Club Guessing since, as is easy to see, it implies the failure of Very Weak Club Guessing. Measuring follows from the Mapping Reflection Principle (MRP), and therefore from PFA, and it can be forced over any model of ZFC.

From Measuring as a vantage point, one can attempt to consider even stronger failures of Club Guessing. In this vein, the following parametrized family of strengthenings of Measuring was considered in [2].

Definition. For a cardinal $\kappa$, let Measuring ${ }_{<\kappa}$ denote the statement that whenever $\overrightarrow{\mathcal{C}}=\left\langle\mathcal{C}_{\alpha}: \alpha \in \omega_{1} \cap \operatorname{Lim}\right\rangle$ is a sequence such that each $\mathcal{C}_{\alpha}$ is a family of fewer than $\kappa$ many closed subsets of $\alpha$, there exists a club $D \subseteq \omega_{1}$ with the property that for every limit point $\delta$ of $D$ and every $c \in \mathcal{C}_{\delta}, D \cap \delta$ measures $c$. For a cardinal $\lambda$, let Measuring $_{\lambda}$ denote Measuring $<_{\lambda^{+}}$.

In the situation given by the above definition, we say that $D$ measures $\overrightarrow{\mathcal{C}}$. We also define Strong Measuring to be the statement Measuring $<2_{2 \omega}$.

In the present article we contribute to the body of information on Measuring and related strong failures of Club Guessing (see also [8], 3], [5], 6], and [2]). One of the questions left unresolved in [2] is whether Measuring $\omega_{1}$ is consistent at all. Answering this question was the motivation for the work in the present article. Our main result is that Strong Measuring $+\neg \mathrm{CH}$ is consistent. In fact, this statement follows from MRP + Martin's Axiom for the class of $\sigma$-centered posets, and also from BPFA 1 We also show the failure, in ZFC, of Measuring ${ }_{\kappa}$, where $\kappa$ is among some of the classical cardinal characteristics of the continuum. Finally, we consider very strong versions of Measuring in contexts in which the Axiom of Choice fails.

\section{BACKGROUND}

We review some background material and notation which is needed for understanding the paper. Let $\mathfrak{c}$ denote the cardinality of the continuum $2^{\omega}$. A set $S \subseteq[\omega]^{\omega}$ is a splitting family if for any infinite set $x \subseteq \omega$, there exists $A \in S$ such that $A$ splits $x$ in the sense that both $x \cap A$ and $x \backslash A$ are infinite. The splitting number $\mathfrak{s}$ is the least cardinality of some splitting family. Given functions $f, g: \omega \rightarrow \omega$, we say that $g$ dominates $f$ if for all $n<\omega, f(n)<g(n)$. We say that $g$ eventually dominates $f$ if there is some $m<\omega$ such that $f(n)<g(n)$ for all $n>m$. A family $B \subseteq \omega^{\omega}$ is bounded if there exists a function $g \in \omega^{\omega}$ which eventually dominates every member of $B$, and otherwise it is unbounded. The bounding number $\mathfrak{b}$ is the least cardinality of some unbounded family. Both cardinal characteristics $\mathfrak{s}$ and $\mathfrak{b}$ are uncountable.

Let $\mathbb{P}$ be a forcing poset. A set $X \subseteq \mathbb{P}$ is centered if every finite subset of $X$ has a lower bound. We say that $\mathbb{P}$ is $\sigma$-centered if it is a union of countably many centered sets. Martin's Axiom for $\sigma$-centered forcings (MA( $\sigma$-centered)) is the statement that for any $\sigma$-centered forcing $\mathbb{P}$ and any collection of fewer than $\mathfrak{c}$ many dense subsets of $\mathbb{P}$, there exists a filter on $\mathbb{P}$ which meets each dense set in the collection. More generally, let $\mathfrak{m}(\sigma$-centered) be the least cardinality of a collection of dense subsets of some $\sigma$-centered forcing poset for which there does not exist a

\footnotetext{
${ }^{1}$ We can also prove the consistency of Strong Measuring with the continuum being arbitrarily large. This result will appear in a sequel to the present article.
} 
filter which meets each dense set in the collection. Note that $\mathrm{MA}(\sigma-$-centered $)$ is equivalent to the statement that $\mathfrak{m}(\sigma$-centered) equals $\mathfrak{c}$.

The Bounded Proper Forcing Axiom (BPFA) is the statement that whenever $\mathbb{P}$ is a proper forcing and $\left\langle A_{i}: i<\omega_{1}\right\rangle$ is a sequence of maximal antichains of $\mathbb{P}$ each of size at most $\omega_{1}$, then there exists a filter on $\mathbb{P}$ which meets each $A_{i}(\underline{9})$ ). We note that BPFA implies $\mathfrak{c}=\omega_{2}([12$, Section 5]). It easily follows that BPFA implies Martin's Axiom, and in particular, implies MA $(\sigma$-centered $)$. The forcing axiom BPFA is equivalent to the statement that for any proper forcing poset $\mathbb{P}$ and any $\Sigma_{1}$ statement $\Phi$ with a parameter from $H\left(\omega_{2}\right)$, if $\Phi$ holds in a generic extension by $\mathbb{P}$, then $\Phi$ holds in the ground model ([7]).

An open stationary set mapping for an uncountable set $X$ and regular cardinal $\theta>\omega_{1}$ is a function $\Sigma$ whose domain is the collection of all countable elementary substructures $M$ of $H(\theta)$ with $X \in M$, such that for all such $M, \Sigma(M)$ is an open, $M$-stationary subset of $[X]^{\omega}$. By open we mean in the Ellentuck topology on $[X]^{\omega}$, and $M$-stationary means meeting every club subset of $[X]^{\omega}$ which is a member of $M$ (see 12 for the complete details). In this article, we are only concerned with these ideas in the simplest case that $X=\omega_{1}$ and for each $M \in \operatorname{dom}(\Sigma), \Sigma(M) \subseteq \omega_{1}$. In this case, being open is equivalent to being open in the topology on $\omega_{1}$ with basis the collection of all open intervals of ordinals, and being $M$-stationary is equivalent to meeting every club subset of $\omega_{1}$ in $M$.

For an open stationary set mapping $\Sigma$ for $X$ and $\theta$, a $\Sigma$-reflecting sequence is an $\in$-increasing and continuous sequence $\left\langle M_{i}: i<\omega_{1}\right\rangle$ of countable elementary substructures of $H(\theta)$ containing $X$ as a member satisfying that for all limit ordinals $\delta<\omega_{1}$, there exists $\beta<\delta$ so that for all $\beta \leq \xi<\delta, M_{\xi} \cap X \in \Sigma\left(M_{\delta}\right)$. The Mapping Reflection Principle (MRP) is the statement that for any open stationary set mapping $\Sigma$, there exists a $\Sigma$-reflecting sequence. We will use the fact that for any open stationary set mapping $\Sigma$, there exists a proper forcing which adds a $\Sigma$-reflecting sequence ([12, Section 3]). Consequently, MRP follows from PFA.

\section{Parametrized Measuring and Club Guessing}

Let $X$ and $Y$ be countable subsets of $\omega_{1}$ with the same supremum $\delta$. We say that $X$ measures $Y$ if there exists $\beta<\delta$ such that $X \backslash \beta$ is either contained in, or disjoint from, $Y$. Measuring is the statement that for any sequence $\left\langle c_{\alpha}: \alpha \in \omega_{1} \cap \operatorname{Lim}\right\rangle$, where each $c_{\alpha}$ is a closed and cofinal subset of $\alpha$, there exists a club $D \subseteq \omega_{1}$ such that for all limit points $\alpha$ of $D, D \cap \alpha$ measures $c_{\alpha}$.

The next two results are due to J. T. Moore ([8]).

Theorem 2.1. MRP implies Measuring.

Theorem 2.2. BPFA implies Measuring.

We now describe parametrized forms of measuring which were introduced in [2]. Let $\overrightarrow{\mathcal{C}}=\left\langle\mathcal{C}_{\alpha}: \alpha \in \omega_{1} \cap \operatorname{Lim}\right\rangle$ be a sequence such that each $\mathcal{C}_{\alpha}$ is a collection of closed and cofinal subsets of $\alpha$. A club $D \subseteq \omega_{1}$ is said to measure $\overrightarrow{\mathcal{C}}$ if for all $\alpha \in \lim (D)$ and all $c \in \mathcal{C}_{\alpha}, D \cap \alpha$ measures $c$.

Definition 2.3. For a cardinal $\kappa$, let Measuring $<_{\kappa}$ denote the statement that whenever $\overrightarrow{\mathcal{C}}=\left\langle\mathcal{C}_{\alpha}: \alpha \in \omega_{1} \cap\right.$ Lim $\rangle$ is a sequence such that each $\mathcal{C}_{\alpha}$ is a collection of fewer than $\kappa$ many closed and cofinal subsets of $\alpha$, then there exists a club $D \subseteq \omega_{1}$ which measures $\overrightarrow{\mathcal{C}}$. For a cardinal $\lambda$, let Measuring ${ }_{\lambda}$ denote Measuring $<_{<\lambda^{+}}$. 
Observe that the principle Measuring is the same as Measuring ${ }_{1}$. If $\kappa<\lambda$, then clearly Measuring $<\lambda$ implies Measuring ${ }_{<\kappa}$. It is easy to see that Measuring $g_{\mathfrak{c}}$ is false.

Definition 2.4. Strong Measuring is the statement that Measuring $<_{<\mathfrak{c}}$ holds.

Since the intersection of countably many clubs in $\omega_{1}$ is club, Measuring easily implies Measuring $_{\omega}$. In particular, Measuring together with $\mathrm{CH}$ implies Strong Measuring. We will prove in Section 3 the consistency of Strong Measuring together with $\neg \mathrm{CH}$. We also observe at the end of that section that Measuring does not imply Measuring $_{\omega_{1}}$.

Proposition 2.5 (2]). Measuring $\mathfrak{s}_{\mathfrak{s}}$ is false.

Proof. Fix a splitting family $S$ of cardinality $\mathfrak{s}$. For each limit ordinal $\alpha<\omega_{1}$, fix a function $f_{\alpha}: \omega \rightarrow \alpha$ which is increasing and cofinal in $\alpha$. For each $A \in S$, let $c_{\alpha, A}=\bigcup\left\{\left(f_{\alpha}(n), f_{\alpha}(n+1)\right]: n \in A\right\}$, which is clearly closed and cofinal in $\alpha$. Let $\mathcal{C}_{\alpha}:=\left\{c_{\alpha, A}: A \in S\right\}$. Then $\overrightarrow{\mathcal{C}}:=\left\langle\mathcal{C}_{\alpha}: \alpha \in \omega_{1} \cap \operatorname{Lim}\right\rangle$ is a sequence such that for each $\alpha, \mathcal{C}_{\alpha}$ is a collection of at most $\mathfrak{s}$ many closed and cofinal subsets of $\alpha$.

Let $D \subseteq \omega_{1}$ be a club. Fix $\alpha \in \lim (D)$. We will show that there exists a member of $\mathcal{C}_{\alpha}$ which $D \cap \alpha$ does not measure. Define $x:=\left\{n<\omega: D \cap\left(f_{\alpha}(n), f_{\alpha}(n+1)\right] \neq\right.$ $\emptyset\}$. Since $\alpha \in \lim (D), x$ is infinite. As $S$ is a splitting family, we can fix $A \in S$ which splits $x$. So both $x \cap A$ and $x \backslash A$ are infinite. We claim that $D \cap \alpha$ does not measure $c_{\alpha, A}$.

Suppose for a contradiction that for some $\beta<\alpha,(D \cap \alpha) \backslash \beta$ is either a subset of, or disjoint from, $c_{\alpha, A}$. Since $A \cap x$ is infinite, we can fix $n \in A \cap x$ such that $f_{\alpha}(n)>\beta$. Then $n \in x$ implies that $D \cap\left(f_{\alpha}(n), f_{\alpha}(n+1)\right] \neq \emptyset$, and $n \in A$ implies that $\left(f_{\alpha}(n), f_{\alpha}(n+1)\right] \subseteq c_{\alpha, A}$. It follows that $(D \cap \alpha) \backslash \beta$ meets $c_{\alpha, A}$. By the choice of $\beta$, this implies that $(D \cap \alpha) \backslash \beta$ is a subset of $c_{\alpha, A}$. But $x \backslash A$ is also infinite, so we can fix $m \in x \backslash A$ such that $f_{\alpha}(m)>\beta$. Then $m \in x$ implies that $D \cap\left(f_{\alpha}(m), f_{\alpha}(m+1)\right] \neq \emptyset$, and $m \notin A$ implies that $\left(f_{\alpha}(m), f_{\alpha}(m+1)\right]$ is disjoint from $c_{\alpha, A}$. Thus, there is a member of $(D \cap \alpha) \backslash \beta$ which is not in $c_{\alpha, A}$, which is a contradiction.

We will prove later in this section that Measuring $\mathfrak{b}$ is also false.

We now turn to parametrized club guessing. We recall some standard definitions. Consider a sequence $\vec{L}=\left\langle L_{\alpha}: \alpha \in \omega_{1} \cap \operatorname{Lim}\right\rangle$, where each $L_{\alpha}$ is a cofinal subset of $\alpha$ with order type $\omega$ (that is, a ladder system). We say that $\vec{L}$ is a club guessing sequence, weak club guessing sequence, or very weak club guessing sequence, respectively, if for every club $D \subseteq \omega_{1}$, there exists a limit ordinal $\alpha<\omega_{1}$ such that:

(1) $L_{\alpha} \subseteq D$,

(2) $L_{\alpha} \backslash D$ is finite, or

(3) $L_{\alpha} \cap D$ is infinite, respectively.

We say that Club Guessing, Weak Club Guessing, or Very Weak Club Guessing holds, respectively, if there exists a club guessing sequence, a weak club guessing sequence, or a very weak club guessing sequence, respectively. It is well known that Measuring implies the failure of Very Weak Club Guessing (see Proposition 2.8 below).

Definition 2.6. Let $\overrightarrow{\mathcal{L}}=\left\langle\mathcal{L}_{\alpha}: \alpha \in \omega_{1} \cap \operatorname{Lim}\right\rangle$ be a sequence where each $\mathcal{L}_{\alpha}$ is a non-empty collection of cofinal subsets of $\alpha$ with order type $\omega$. The sequence $\overrightarrow{\mathcal{L}}$ is said to be a club guessing sequence, weak club guessing sequence, or very weak 
club guessing sequence, respectively, if for every club $D \subseteq \omega_{1}$, there exists a limit ordinal $\alpha<\omega_{1}$ and some $L \in \mathcal{L}_{\alpha}$ such that:

(1) $L \subseteq D$,

(2) $L \backslash D$ is finite, or

(3) $L \cap D$ is infinite, respectively.

Definition 2.7. For a cardinal $\kappa$, let $C G_{<\kappa}, W C G_{<\kappa}$, and $V W C G_{<\kappa}$, respectively, be the statements that there exists a club guessing sequence, weak club guessing sequence, or very weak club guessing sequence $\left\langle\mathcal{L}_{\alpha}: \alpha \in \omega_{1} \cap \operatorname{Lim}\right\rangle$, respectively, such that for each $\alpha,\left|\mathcal{L}_{\alpha}\right|<\kappa$. Let $C G_{\kappa}, W C G_{\kappa}$, and $V W C G_{\kappa}$ denote the statements $C G_{<\kappa^{+}}, W C G_{<\kappa^{+}}$, and $V W C G_{<\kappa^{+}}$, respectively.

Clearly, if $\kappa<\lambda$, then $\mathrm{CG}_{<\kappa}$ implies $\mathrm{CG}_{<\lambda}$, and similarly with WCG and VWCG. Observe that Club Guessing, Weak Club Guessing, and Very Weak Club Guessing are equivalent to $C_{1}, W C G_{1}$, and $V W C G_{1}$, respectively. Obviously, $C_{c}$ is true. The weakest forms of club guessing principles which are not provable in ZFC are when the index is $<\mathfrak{c}$.

Proposition 2.8. For any cardinal $\kappa \geq 2$, Measuring $<\kappa$ implies the failure of $V W C G_{<\kappa}$.

Proof. Suppose for a contradiction that Measuring ${ }_{<\kappa}$ and $\mathrm{VWCG}_{<\kappa}$ both hold. Fix a very weak club guessing sequence $\overrightarrow{\mathcal{L}}=\left\langle\mathcal{L}_{\alpha}: \alpha \in \omega_{1} \cap \operatorname{Lim}\right\rangle$ such that each $\mathcal{L}_{\alpha}$ has cardinality less than $\kappa$. Observe that for each $\alpha$, every member of $\mathcal{L}_{\alpha}$ is vacuously a closed subset of $\alpha$ since it has order type $\omega$.

By Measuring $_{<\kappa}$, there exists a club $D \subseteq \omega_{1}$ which measures $\overrightarrow{\mathcal{L}}$. Let $E$ be the club set of indecomposable limit ordinals $\alpha>\omega$ in $\lim (D)$ such that $\operatorname{ot}(D \cap \alpha)=\alpha$. Since $\overrightarrow{\mathcal{L}}$ is a very weak club guessing sequence, there exists a limit ordinal $\alpha$ and $L \in \mathcal{L}_{\alpha}$ such that $L \cap E$ is infinite. In particular, $\alpha$ is a limit point of $E$, and hence of $D$.

Since $D$ measures $\overrightarrow{\mathcal{L}}$ and $L \in \mathcal{L}_{\alpha}, D \cap \alpha$ measures $L$. So we can fix $\beta<\alpha$ such that $(D \cap \alpha) \backslash \beta$ is either a subset of, or disjoint from, $L$. Now $L \cap E$, and hence $L \cap D$, is infinite. As $L$ has order type $\omega$, this implies that $L \cap D$ is cofinal in $\alpha$. By the choice of $\beta,(D \cap \alpha) \backslash \beta$ must be a subset of $L$. But since $\alpha \in E \operatorname{ot}(D \cap \alpha)=\alpha$ and $\alpha$ is indecomposable, which implies that ot $((D \cap \alpha) \backslash \beta)=\alpha$. As $\alpha>\omega$, this is impossible since $(D \cap \alpha) \backslash \beta$ is a subset of $L$ and $L$ has order type $\omega$.

In particular, since Strong Measuring is consistent, so is the failure of $\mathrm{VWCG}_{<\mathfrak{c}}$. (The consistency of $\neg \mathbf{V W C G} G_{<\mathfrak{c}}$ together with $\mathfrak{c}$ arbitrarily large was previously shown in 4.)

Proposition 2.9 (Hrušák [5]). VWCG $G_{\mathfrak{b}}$ is true.

Proof. Fix an unbounded family $\left\{r_{\alpha}: \alpha<\mathfrak{b}\right\}$ in $\omega^{\omega}$. For each limit ordinal $\delta<\omega_{1}$, fix a cofinal subset $C_{\delta}$ of $\delta$ with order type $\omega$ and a bijection $h_{\delta}: \omega \rightarrow \delta$. Let $C_{\delta}(n)$ denote the $n$-th member of $C_{\delta}$ for all $n<\omega$. For all limit ordinals $\delta<\omega_{1}$ and $\alpha<\mathfrak{b}$, define

$$
A_{\delta}^{\alpha}:=C_{\delta} \cup \bigcup\left\{h_{\delta}\left[r_{\alpha}(n)\right] \backslash C_{\delta}(n): n<\omega\right\} .
$$

It is easy to check that for all $\delta$ and $\alpha, A_{\delta}^{\alpha}$ has order type $\omega$ and $\sup \left(A_{\delta}^{\alpha}\right)=\delta$. Given a club $C \subseteq \omega_{1}$, let $\delta$ be a limit point of $C$ and let $g_{C, \delta}: \omega \rightarrow \omega$ be the function given by

$$
g_{C, \delta}(n)=\min \left\{m<\omega: h_{\delta}(m) \in C \backslash C_{\delta}(n)\right\} .
$$


Now let $\alpha<\mathfrak{b}$ be such that $r_{\alpha}(n)>g_{C, \delta}(n)$ for infinitely many $n$. It then follows that $\left|A_{\delta}^{\alpha} \cap C\right|=\omega$.

By Propositions 2.8 and 2.9, the following is immediate.

Corollary 2.10. Measuring $\mathfrak{b}_{\mathfrak{b}}$ is false.

An obvious question is whether the parametrized versions of club guessing are actually the same as the usual ones. We conclude this section by showing that they are not.

Recall that a forcing poset $\mathbb{P}$ is $\omega^{\omega}$-bounding if every function in $\omega^{\omega} \cap V^{\mathbb{P}}$ is dominated by a function in $\omega^{\omega} \cap V$.

Lemma 2.11 (Hrušák). Assume that VWCG fails. Let $\mathbb{P}$ be any $\omega_{1}-$ c.c., $\omega^{\omega}$ bounding forcing. Then $\mathbb{P}$ forces that VWCG fails.

Proof. Since $\mathbb{P}$ is $\omega_{1}$-c.c. and $\omega^{\omega}$-bounding, a standard argument shows that whenever $p \in \mathbb{P}$ and $p$ forces that $\dot{b} \in \omega^{\omega}$, then there exists a function $b^{*} \in \omega^{\omega}$ such that $p$ forces that $b^{*}$ dominates $\dot{b}$.

Let us show that whenever $p \in \mathbb{P}, \delta<\omega_{1}$, and $p$ forces that $\dot{X}$ is a cofinal subset of $\delta$ of order type $\omega$, then there exists a set $Y$ with order type $\omega$ such that $p$ forces that $\dot{X} \subseteq Y$. To see this, fix a bijection $f: \omega \rightarrow \delta$ and a strictly increasing sequence $\left\langle\alpha_{n}: n<\omega\right\rangle$ cofinal in $\alpha$ with $\alpha_{0}=0$. We claim that there exists a $\mathbb{P}$-name $\dot{b}$ for a function from $\omega$ to $\omega$ such that $p$ forces that for all $n<\omega, \dot{b}(n)$ is the least $m<\omega$ such that $\dot{X} \cap\left[\alpha_{n}, \alpha_{n+1}\right) \subseteq f[m]$. This is true since $p$ forces that $\dot{X}$ has order type $\omega$ and hence that $\dot{X} \cap\left[\alpha_{n}, \alpha_{n+1}\right)$ is finite for all $n<\omega$. Fix a function $b^{*}: \omega \rightarrow \omega$ such that $p$ forces that $b^{*}$ dominates $\dot{b}$. Now let

$$
Y:=\bigcup\left\{f\left[b^{*}(n)\right] \cap\left[\alpha_{n}, \alpha_{n+1}\right): n<\omega\right\} .
$$

It is easy to check that $Y$ has order type $\omega$ and $p$ forces that $\dot{X} \subseteq Y$.

Now we are ready to prove the proposition. So suppose that $p \in \mathbb{P}$ forces that $\left\langle\dot{X}_{\alpha}: \alpha \in \omega_{1} \cap \operatorname{Lim}\right\rangle$ is a very weak club guessing sequence. By the previous paragraph, for each limit ordinal $\alpha<\omega_{1}$ we can fix a cofinal subset $Y_{\alpha}$ of $\alpha$ with order type $\omega$ such that $p$ forces that $\dot{X}_{\alpha} \subseteq Y_{\alpha}$. We claim that $\left\langle Y_{\alpha}: \alpha \in \omega_{1} \cap \operatorname{Lim}\right\rangle$ is a very weak club guessing sequence in the ground model, which completes the proof. So consider a club $C \subseteq \omega_{1}$. Then $C$ is still a club in $V^{\mathbb{P}}$. Fix $q \leq p$ and a limit ordinal $\alpha<\omega_{1}$ such that $q$ forces that $\dot{X}_{\alpha} \cap C$ is infinite. Then clearly $q$ forces that $Y_{\alpha} \cap C$ is infinite, so in fact, $Y_{\alpha} \cap C$ is infinite.

Proposition 2.12. It is consistent that $\neg V W C G$ and $C G_{\omega_{1}}$ both hold.

Proof. Let $V$ be a model in which $\mathrm{CH}$ holds and VWCG fails. Such a model was shown to exist by Shelah [13]. Let $\mathbb{P}$ be an $\omega_{1}$-c.c., $\omega^{\omega}$-bounding forcing poset which adds at least $\omega_{2}$ many reals; for example, random real forcing with product measure is such a forcing. We claim that in $V^{\mathbb{P}}, C_{\omega_{1}}$ holds but VWCG fails. By Lemma 2.11, VWCG is false in $V^{\mathbb{P}}$. In $V$, define $\overrightarrow{\mathcal{L}}=\left\langle\mathcal{L}_{\alpha}: \alpha \in \omega_{1} \cap\right.$ Lim $\rangle$ by letting $\mathcal{L}_{\alpha}$ be the collection of all cofinal subsets of $\alpha$ with order type $\omega$. Since $\mathrm{CH}$ holds, the cardinality of each $\mathcal{L}_{\alpha}$ is $\omega_{1}$. If $C$ is a club subset of $\omega_{1}$ in $V^{\mathbb{P}}$, then since $\mathbb{P}$ is $\omega_{1}$-c.c., there is a club $D \subseteq \omega_{1}$ in $V$ such that $D \subseteq C$. In $V$, fix $d \subseteq D$ with order type $\omega$, and let $\alpha:=\sup (d)$. Then $d \in \mathcal{L}_{\alpha}$ and $d \subseteq C$. Thus, $\overrightarrow{\mathcal{L}}$ witnesses that $\mathrm{CG}_{\omega_{1}}$ holds in $V^{\mathbb{P}}$. 


\section{The Consistency of Strong Measuring And $\neg \mathrm{CH}$}

As we previously mentioned, Measuring is equivalent to Measuring $\omega$, and therefore under $\mathrm{CH}$, Measuring is equivalent to Strong Measuring. In this section we establish the consistency of Strong Measuring with the negation of $\mathrm{CH}$. More precisely, we will prove that MRP together with MA( $\sigma$-centered $)$ implies Strong Measuring, and BPFA implies Strong Measuring. Recall that both MRP and BPFA imply that $\mathfrak{c}=\omega_{2}([12)$.

A set $M$ is suitable if for some regular cardinal $\theta>\omega_{1}, M$ is a countable elementary substructure of $H(\theta)$. We will follow the conventions introduced in Section 1 that the properties "open" and " $M$-stationary" refer to open and $M$ stationary subsets of $\omega_{1}$ (where $\omega_{1}$ is considered as a subspace of $\left[\omega_{1}\right]^{\omega}$ ).

Proposition 3.1. Assume that $M$ is suitable. Let $\delta:=M \cap \omega_{1}$. Suppose that $\mathcal{Y}$ is a collection of open subsets of $\delta$ such that for any finite set $a \subseteq \mathcal{Y}, \cap a$ is $M$-stationary. Then there exists a $\sigma$-centered forcing $\mathbb{P}$ and a collection $\mathcal{D}$ of dense subsets of $\mathbb{P}$ of size at most $|\mathcal{Y}|+\omega$ such that whenever $G$ is a filter on $\mathbb{P}$ in some outer model $W$ of $V$ with $\omega_{1}^{V}=\omega_{1}^{W}$ which meets each member of $\mathcal{D}$, then there exists a set $z \subseteq \delta$ in $W$ which is open, $M$-stationary, and satisfies that for all $X \in \mathcal{Y}, z \backslash X$ is bounded in $\delta$.

Proof. Define a forcing poset $\mathbb{P}$ to consist of conditions which are pairs $(x, a)$, where $x$ is an open and bounded subset of $\delta$ in $M$ and $a$ is a finite subset of $\mathcal{Y}$. Let $(y, b) \leq(x, a)$ if $y$ is an end-extension of $x, a \subseteq b$, and $y \backslash x \subseteq \bigcap a$.

Since $M$ is countable, there are only countably many possibilities for the first component of a condition. If $\left(x, a_{0}\right), \ldots,\left(x, a_{n}\right)$ are finitely many conditions with the same first component, then easily $\left(x, a_{0} \cup \ldots \cup a_{n}\right)$ is a condition in $\mathbb{P}$ which is below each of the conditions $\left(x, a_{0}\right), \ldots,\left(x, a_{n}\right)$. It follows that $\mathbb{P}$ is $\sigma$-centered.

For each $X \in \mathcal{Y}$, let $D_{X}$ denote the set of conditions $(x, a)$ such that $X \in a$. Observe that $D_{X}$ is dense. For every club $C$ of $\omega_{1}$ which is a member of $M$, let $E_{C}$ denote the set of conditions $(x, a)$ such that $x \cap C$ is non-empty. We claim that $E_{C}$ is dense. Let $(x, a)$ be a condition. Since $\bigcap a$ is $M$-stationary and $\lim (C) \backslash(\sup (x)+1)$ is a club subset of $\omega_{1}$ in $M$, we can find a limit ordinal $\alpha$ in $C \cap(\bigcap a)$ which is in the interval $(\sup (x), \delta)$. Since $\alpha \in \bigcap a$ and $\bigcap a$ is open, we can find $\beta<\gamma<\delta$ such that $\alpha \in(\beta, \gamma) \subseteq \bigcap a$. As $\sup (x)+1<\alpha$, without loss of generality $\sup (x)<\beta$. By elementarity, the interval $b:=(\beta, \gamma)$ is in $M$. It follows that $(x \cup b, a)$ is a condition, $x \cup b$ end-extends $x$, and $(x \cup b) \backslash x=b \subseteq \bigcap a$. Thus, $(x \cup b, a) \leq(x, a)$, and since $\alpha \in C,(x \cup b, a) \in E_{C}$.

Let $\mathcal{D}$ denote the collection of all dense sets of the form $D_{X}$ where $X \in \mathcal{Y}$, or $E_{C}$ where $C$ is a club subset of $\omega_{1}$ belonging to $M$. Then $|\mathcal{D}| \leq|\mathcal{Y}|+\omega$. Let $G$ be a filter on $\mathbb{P}$ in some outer model $W$ with $\omega_{1}^{V}=\omega_{1}^{W}$ which meets each dense set in $\mathcal{D}$. Define $z:=\bigcup\{x: \exists a(x, a) \in G\}$. Note that since $z$ is a union of open sets, it is open (using the fact that being open is absolute between $V$ and $W$ ). For each club $C \subseteq \omega_{1}$ which lies in $M$, there exists a condition $(x, a)$ which belongs to $G \cap E_{C}$, and thus $x \cap C \neq \emptyset$. Therefore, $z \cap C \neq \emptyset$. Hence, $z$ is $M$-stationary.

It remains to show that for all $X \in \mathcal{Y}, z \backslash X$ is bounded in $\delta$. Consider $X \in \mathcal{Y}$. Then we can fix $(x, a) \in G \cap D_{X}$, which means that $X \in a$. Now the definition of the ordering on $\mathbb{P}$ together with the fact that $G$ is a filter easily implies that $z \backslash x \subseteq X$. Therefore, $z \backslash X \subseteq x$, and hence $z \backslash X$ is bounded in $\delta$. 
Corollary 3.2. Assume that $M$ is suitable. Let $\delta:=M \cap \omega_{1}$. Suppose that $\mathcal{Y}$ is a collection of less than $\mathfrak{m}(\sigma$-centered) many open subsets of $\delta$ such that for any finite set $a \subseteq \mathcal{Y}, \cap a$ is $M$-stationary. Then there exists a set $z \subseteq \delta$ which is open, $M$-stationary, and satisfies that for all $X \in \mathcal{Y}, z \backslash X$ is bounded in $\delta$.

Proof. Fix a $\sigma$-centered forcing $\mathbb{P}$ and a collection $\mathcal{D}$ of dense subsets of $\mathbb{P}$ of size at most $|\mathcal{Y}|+\omega$ as described in Proposition 3.1. Since $\mathfrak{m}(\sigma$-centered) is uncountable, $|\mathcal{D}|<\mathfrak{m}(\sigma$-centered $)$. Hence, there exists a filter $G$ on $\mathbb{P}$ which meets each dense set in $\mathcal{D}$. By Proposition 3.1, there exists a set $z \subseteq \delta$ which is open, $M$-stationary, and satisfies that for all $X \in \mathcal{Y}, z \backslash X$ is bounded in $\delta$.

Proposition 3.3. Let $\overrightarrow{\mathcal{C}}=\left\langle\mathcal{C}_{\alpha}: \alpha \in \omega_{1} \cap\right.$ Lim $\rangle$ be a sequence such that each $\mathcal{C}_{\alpha}$ is a collection of less than $\mathfrak{m}(\sigma$-centered) many closed and cofinal subsets of $\alpha$. Then there exists an open stationary set mapping $\Sigma$ such that, if $W$ is any outer model with the same $\omega_{1}$ in which there exists a $\Sigma$-reflecting sequence, then there exists in $W$ a club subset of $\omega_{1}$ which measures $\overrightarrow{\mathcal{C}}$.

Proof. For each limit ordinal $\alpha<\omega_{1}$, let $\mathcal{D}_{\alpha}:=\left\{\alpha \backslash c: c \in \mathcal{C}_{\alpha}\right\}$. Observe that each $\mathcal{D}_{\alpha}$ is a collection of fewer than $\mathfrak{m}(\sigma$-centered) many open subsets of $\alpha$.

We will define $\Sigma$ to have domain the collection of all countable elementary substructures $M$ of $H\left(\omega_{2}\right)$. Consider such an $M$ and we define $\Sigma(M)$. Note that $M$ is suitable. Let $\delta:=M \cap \omega_{1}$. We consider two cases. In the first case, there does not exist a member of $\mathcal{D}_{\delta}$ which is $M$-stationary. Define $\Sigma(M)=\delta$, which is clearly open and $M$-stationary.

In the second case, there exists some member of $\mathcal{D}_{\delta}$ which is $M$-stationary. A straightforward application of Zorn's lemma implies that there exists a nonempty set $\mathcal{Y}_{M} \subseteq \mathcal{D}_{\delta}$ such that for any $a \in\left[\mathcal{Y}_{M}\right]^{<\omega}, \bigcap a$ is $M$-stationary, and moreover, $\mathcal{Y}_{M}$ is a maximal subset of $\mathcal{D}_{\delta}$ with this property. Since $\mathcal{Y}_{M} \subseteq \mathcal{D}_{\delta}$, $\left|\mathcal{Y}_{M}\right|<\mathfrak{m}(\sigma$-centered $)$. So the collection $\mathcal{Y}_{M}$ satisfies the assumptions of Corollary 3.2. It follows that there exists a set $z_{M} \subseteq \delta$ which is open, $M$-stationary, and satisfies that for all $X \in \mathcal{Y}_{M}, z_{M} \backslash X$ is bounded in $\delta$. Now define $\Sigma(M):=z_{M}$.

This completes the definition of $\Sigma$. Consider an outer model $W$ of $V$ with the same $\omega_{1}$, and assume that in $W$ there exists a $\Sigma$-reflecting sequence $\left\langle M_{\delta}: \delta<\omega_{1}\right\rangle$. Let $\alpha_{\delta}:=M_{\delta} \cap \omega_{1}$ for all $\delta<\omega_{1}$. Let $D$ be the club set of $\delta<\omega_{1}$ such that $\alpha_{\delta}=\delta$. We claim that $D$ measures $\overrightarrow{\mathcal{C}}$.

Consider $\delta \in \lim (D)$. Then $\delta=\alpha_{\delta}=M_{\delta} \cap \omega_{1}$. Let $M:=M_{\delta}$. We first claim that if $c \in \mathcal{C}_{\delta}$ and $\delta \backslash c$ is not $M$-stationary, then for some $\beta<\delta,(D \cap \delta) \backslash \beta \subseteq c$. Fix a club subset $E$ of $\omega_{1}$ in $M$ which is disjoint from $\delta \backslash c$. By the continuity of the $\Sigma$-reflecting sequence, there exists $\beta<\delta$ such that $E \in M_{\beta}$. We claim that $(D \cap \delta) \backslash \beta \subseteq c$. Let $\xi \in(D \cap \delta) \backslash \beta$. Then $E \in M_{\xi}$, and hence by elementarity, $\xi=M_{\xi} \cap \omega_{1} \in E$. Since $E$ is disjoint from $\delta \backslash c, \xi \in c$.

We split the argument according to the two cases in the definition of $\Sigma(M)$. In the first case, there does not exist a member of $\mathcal{D}_{\delta}$ which is $M$-stationary. Consider $c \in \mathcal{C}_{\delta}$. Then $\delta \backslash c$ is not $M$-stationary. By the previous paragraph, there exists $\beta<\delta$ such that $(D \cap \delta) \backslash \beta \subseteq c$.

In the second case, there exists a member of $\mathcal{D}_{\delta}$ which is $M$-stationary. Consider $c \in \mathcal{C}_{\delta}$. Then $X:=\delta \backslash c \in \mathcal{D}_{\delta}$. We consider two possibilities. First, assume that $X$ is in $\mathcal{Y}_{M}$. By the choice of $\mathcal{Y}_{M}$ and $z_{M}$, we know that $z_{M} \backslash X$ is bounded in $\delta$. So fix $\beta_{0}<\delta$ so that $z_{M} \backslash \beta_{0} \subseteq X$. By the definition of being a $\Sigma$-reflecting sequence, there exists $\beta_{1}<\delta$ so that for all $\beta_{1} \leq \xi<\delta, M_{\xi} \cap \omega_{1} \in \Sigma(M)=z_{M}$. 
Let $\beta:=\max \left\{\beta_{1}, \beta_{2}\right\}$. Consider $\xi \in(D \cap \delta) \backslash \beta$. Then $\xi \geq \beta_{1}$ implies that $\xi=M_{\xi} \cap \omega_{1} \in z_{M}$. So $\xi \in z_{M} \backslash \beta_{0} \subseteq X=\delta \backslash c$.

Secondly, assume that $X$ is not in $\mathcal{Y}_{M}$. By the maximality of $\mathcal{Y}_{M}$, there exists a set $a \in\left[\mathcal{Y}_{M}\right]^{<\omega}$ such that $X \cap \bigcap a$ is not $M$-stationary. Fix a club $E$ in $M$ which is disjoint from $X \cap \bigcap a$. By the continuity of the $\Sigma$-reflecting sequence, there exists $\beta<\delta$ such that $E \in M_{\beta}$. Consider $\xi \in(D \cap \delta) \backslash \beta$. Then $E \in M_{\xi}$, which implies that $\xi=M_{\xi} \cap \omega_{1} \in E$. Thus, $\xi$ is not in $X \cap \cap a$. On the other hand, letting $a=\left\{X_{0}, \ldots, X_{n}\right\}$, for each $i \leq n$ the previous paragraph implies that there exists $\beta_{i}<\delta$ such that $(D \cap \delta) \backslash \beta_{i} \subseteq X_{i}$. Let $\beta^{*}$ be an ordinal in $\delta$ which is larger than $\beta$ and $\beta_{i}$ for all $i \leq n$. Consider $\xi \in(D \cap \delta) \backslash \beta^{*}$. Then by the choice of $\beta, \xi \notin X \cap \bigcap a$. By the choice of the $\beta_{i}$ 's, $\xi \in \bigcap a$. Therefore, $\xi \notin X=\delta \backslash c$, which means that $\xi \in c$. Thus, $(D \cap \delta) \backslash \beta^{*} \subseteq c$.

Corollary 3.4. Assume MRP and MA( $\sigma$-centered). Then Strong Measuring holds.

Proof. Let $\overrightarrow{\mathcal{C}}=\left\langle\mathcal{C}_{\alpha}: \alpha \in \omega_{1} \cap \operatorname{Lim}\right\rangle$ be a sequence such that each $\mathcal{C}_{\alpha}$ is a collection of fewer than $\mathfrak{c}$ many closed and cofinal subsets of $\alpha$. We claim that there exists a club subset of $\omega_{1}$ which measures $\overrightarrow{\mathcal{C}}$. By $\operatorname{MA}(\sigma$-centered $), \mathfrak{m}(\sigma$-centered $)$ equals $\mathfrak{c}$. So each $\mathcal{C}_{\alpha}$ has size less than $\mathfrak{m}(\sigma$-centered $)$.

By Proposition 3.3, there exists an open stationary set mapping $\Sigma$ such that, if $W$ is any outer model with the same $\omega_{1}$ in which there exists a $\Sigma$-reflecting sequence, then there exists in $W$ a club subset of $\omega_{1}$ which measures $\overrightarrow{\mathcal{C}}$. Applying MRP, there exists a $\Sigma$-reflecting sequence in $V$. Thus, in $V$ there exists a club subset of $\omega_{1}$ which measures $\overrightarrow{\mathcal{C}}$.

Corollary 3.5. Assume BPFA. Then Strong Measuring holds.

Proof. Let $\overrightarrow{\mathcal{C}}=\left\langle\mathcal{C}_{\alpha}: \alpha \in \omega_{1} \cap \operatorname{Lim}\right\rangle$ be a sequence such that each $\mathcal{C}_{\alpha}$ is a collection of fewer than $\mathfrak{c}=\omega_{2}$ many closed and cofinal subsets of $\alpha$. We claim that there exists a club subset of $\omega_{1}$ which measures $\overrightarrow{\mathcal{C}}$. Since $\mathfrak{c}=\omega_{2}, \overrightarrow{\mathcal{C}}$ is a member of $H\left(\omega_{2}\right)$. Thus, the existence of a club subset of $\omega_{2}$ which measures $\overrightarrow{\mathcal{C}}$ is expressible as a $\Sigma_{1}$ statement involving a parameter in $H\left(\omega_{2}\right)$. By BPFA, it suffices to show that there exists a proper forcing which forces that such a club exists.

Now BPFA implies Martin's Axiom, and in particular, that $\mathfrak{m}(\sigma$-centered $)$ is equal to $\mathfrak{c}$. So each $\mathcal{C}_{\alpha}$ has size less than $\mathfrak{m}(\sigma$-centered $)$. By Proposition 3.3, there exists an open stationary set mapping $\Sigma$ such that, if $W$ is any outer model with the same $\omega_{1}$ in which there exists a $\Sigma$-reflecting sequence, then there exists in $W$ a club subset of $\omega_{1}$ which measures $\overrightarrow{\mathcal{C}}$. By [12, Section 3], there exists a proper forcing $\mathbb{P}$ which adds a $\Sigma$-reflecting sequence, so in $V^{\mathbb{P}}$ there is a club subset of $\omega_{1}$ which measures $\overrightarrow{\mathcal{C}}$.

We now sketch a proof that MRP alone does not imply Strong Measuring. In particular, Measuring does not imply Strong Measuring. Start with a model of $\mathbf{C H}$ in which there exists a supercompact cardinal $\kappa$. Construct a forcing iteration $\mathbb{P}$ in the standard way to obtain a model of MRP. To do this, fix a Laver function $f$ : $\kappa \rightarrow V_{\kappa}$. Then define a countable support forcing iteration $\left\langle\mathbb{P}_{\alpha}, \dot{\mathbb{Q}}_{\beta}: \alpha \leq \kappa, \beta<\kappa\right\rangle$ as follows. Given $\mathbb{P}_{\alpha}$, consider $f(\alpha)$. If $f(\alpha)$ happens to be a $\mathbb{P}_{\alpha}$-name for some open stationary set mapping, then let $\dot{\mathbb{Q}}_{\alpha}$ be a $\mathbb{P}_{\alpha}$-name for a proper forcing which

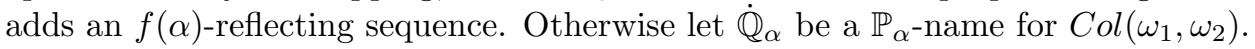
Now define $\mathbb{P}:=\mathbb{P}_{\kappa}$. Arguments similar to those in the standard construction of a model of PFA can be used to show that $\mathbb{P}$ forces MRP. 
The forcing for adding a $\Sigma$-reflecting sequence for a given open stationary set mapping does not add reals ([12, Section 3]). In particular, it is vacuously $\omega^{\omega}{ }_{-}$ bounding. The property of being proper and $\omega^{\omega}$-bounding is preserved under countable support forcing iterations ([1, Theorem 3.5]), so $\mathbb{P}$ is also $\omega^{\omega}$-bounding. In particular, $V \cap \omega^{\omega}$ is an unbounded family in $V^{\mathbb{P}}$, and it has size $\omega_{1}$ since $\mathrm{CH}$ holds in $V$. It follows that the bounding number $\mathfrak{b}$ is equal to $\omega_{1}$. But by Corollary 2.9, Measuring , $_{\mathfrak{b}}$ is false. So $\mathbb{P}$ forces that Measuring $\omega_{1}$ is false. As $\mathfrak{c}=\omega_{2}$ in $V^{\mathbb{P}}$, Strong Measuring fails in $V^{\mathbb{P}}$.

We also note that Strong Measuring plus $\mathfrak{c}=\omega_{2}$ is consistent with the existence of an $\omega_{1}$-Suslin tree. Namely, both the forcing for adding a $\Sigma$-reflecting sequence for a given open stationary set mapping $\Sigma$, as well as any $\sigma$-centered forcing, preserve Suslin trees ([1]). And the property of being proper and preserving a given Suslin tree is preserved under countable support forcings iterations ([10]). So starting with a model in which there exists an $\omega_{1}$-Suslin tree $S$ and a supercompact cardinal $\kappa$, we can iterate forcing similar to the argument in the preceding paragraphs to produce a model of MA $\left(\sigma\right.$-centered) plus MRP in which $S$ is an $\omega_{1}$-Suslin tree. By Corollary 3.4, Strong Measuring holds in that model.

\section{Measuring Without the Axiom of Choice}

Another natural way to strengthen Measuring is to allow, in the sequence to be measured, not just closed sets, but also sets of higher Borel complexity. This line of strengthenings of Measuring was also considered in [2]. For completeness, we are including here the corresponding observations.

The version of Measuring where one considers sequences $\vec{X}=\left\langle X_{\alpha}: \alpha \in \omega_{1} \cap\right.$ $\operatorname{Lim}\rangle$, with each $X_{\alpha}$ an open subset of $\alpha$ in the order topology, is of course equivalent to Measuring. A natural next step would therefore be to consider sequences in which each $X_{\alpha}$ is a countable union of closed sets. This is obviously the same as allowing each $X_{\alpha}$ to be an arbitrary subset of $\alpha$. Let us call the corresponding statement Measuring*:

Definition 4.1. Measuring* holds if and only if for every sequence $\vec{X}=\left\langle X_{\alpha}: \alpha \in\right.$ $\left.\omega_{1} \cap \operatorname{Lim}\right\rangle$, if $X_{\alpha} \subseteq \alpha$ for each $\alpha$, then there is some club $D \subseteq \omega_{1}$ such that for every limit point $\delta \in D$ of $D, D \cap \delta$ measures $X_{\delta}$.

It is easy to see that Measuring* is false in ZFC. In fact, given a stationary and co-stationary $S \subseteq \omega_{1}$, there is no club of $\omega_{1}$ measuring $\vec{X}=\left\langle S \cap \alpha: \alpha \in \omega_{1} \cap \operatorname{Lim}\right\rangle$. The reason is that if $D$ is any club of $\omega_{1}$, then both $D \cap S \cap \delta$ and $(D \cap \delta) \backslash S$ are cofinal subsets of $\delta$ for each $\delta$ in the club of limit points in $\omega_{1}$ of both $D \cap S$ and $D \backslash S$.

The status of Measuring* is more interesting in the absence of the Axiom of Choice. Let $\mathcal{C}_{\omega_{1}}=\left\{X \subseteq \omega_{1}: C \subseteq X\right.$ for some club $C$ of $\left.\omega_{1}\right\}$.

Observation 4.2. (ZF+ $\mathcal{C}_{\omega_{1}}$ is a normal filter on $\left.\omega_{1}\right)$ Suppose $\vec{X}=\left\langle X_{\delta}: \delta \in\right.$ $\left.\omega_{1} \cap \operatorname{Lim}\right\rangle$ is such that

(1) $X_{\delta} \subseteq \delta$ for each $\delta$.

(2) For each club $C \subseteq \omega_{1}$,

(a) there is some $\delta \in C$ such that $C \cap X_{\delta} \neq \emptyset$, and

(b) there is some $\delta \in C$ such that $(C \cap \delta) \backslash X_{\delta} \neq \emptyset$.

Then there is a stationary and co-stationary subset of $\omega_{1}$ definable from $\vec{X}$. 
Proof. We have two possible cases. The first case is that in which for all $\alpha<\omega_{1}$, either

- $W_{\alpha}^{0}=\left\{\delta<\omega_{1}: \alpha \notin X_{\delta}\right\}$ is in $\mathcal{C}_{\omega_{1}}$, or

- $W_{\alpha}^{1}=\left\{\delta<\omega_{1}: \alpha \in X_{\delta}\right\}$ is in $\mathcal{C}_{\omega_{1}}$.

For each $\alpha<\omega_{1}$, let $W_{\alpha}$ be $W_{\alpha}^{\epsilon}$ for the unique $\epsilon \in\{0,1\}$ such that $W_{\alpha}^{\epsilon} \in \mathcal{C}_{\omega_{1}}$, and let $W^{*}=\Delta_{\alpha<\omega_{1}} W_{\alpha} \in \mathcal{C}_{\omega_{1}}$. Then $X_{\delta_{0}}=X_{\delta_{1}} \cap \delta_{0}$ for all $\delta_{0}<\delta_{1}$ in $W^{*}$. It then follows, by (2), that $S=\bigcup_{\delta \in W^{*}} X_{\delta}$, which of course is definable from $\vec{C}$, is a stationary and co-stationary subset of $\omega_{1}$. Indeed, suppose $C \subseteq \omega_{1}$ is a club, and let us fix a club $D \subseteq W^{*}$. There is then some $\delta \in C \cap D$ and some $\alpha \in C \cap D \cap X_{\delta}$. But then $\alpha \in S$ since $\delta \in W^{*}$ and $\alpha \in W^{*} \cap X_{\delta}$. There is also some $\delta \in C \cap D$ and some $\alpha \in C \cap D$ such that $\alpha \notin X_{\delta}$, which implies that $\alpha \notin S$ by a symmetrical argument, using the fact that $X_{\delta_{0}}=X_{\delta_{1}} \cap \delta_{0}$ for all $\delta_{0}<\delta_{1}$ in $W^{*}$.

The second possible case is that there is some $\alpha<\omega_{1}$ with the property that both $W_{\alpha}^{0}$ and $W_{\alpha}^{1}$ are stationary subsets of $\omega_{1}$. But now we can let $S$ be $W_{\alpha}^{0}$, where $\alpha$ is first such that $W_{\alpha}^{0}$ is stationary and co-stationary.

It is worth comparing the above observation with Solovay's classic result that an $\omega_{1}$-sequence of pairwise disjoint stationary subsets of $\omega_{1}$ is definable from any given ladder system on $\omega_{1}$ (working in the same theory).

Corollary 4.3. $\left(Z F+\mathcal{C}_{\omega_{1}}\right.$ is a normal filter on $\left.\omega_{1}\right)$ The following are equivalent.

(1) $\mathcal{C}_{\omega_{1}}$ is an ultrafilter on $\omega_{1}$

(2) Measuring*;

(3) For every sequence $\left\langle X_{\alpha}: \alpha \in \omega_{1} \cap \operatorname{Lim}\right\rangle$, if $X_{\alpha} \subseteq \alpha$ for each $\alpha$, then there is a club $C \subseteq \omega_{1}$ such that either

- $C \cap \delta \subseteq X_{\delta}$ for every $\delta \in C$, or

- $C \cap X_{\delta}=\emptyset$ for every $\delta \in C$.

Proof. (3) trivially implies (2), and by the observation (1) implies (3). Finally, to see that (2) implies (1), note that the argument right after the definition of Measuring* uses only ZF together with the regularity of $\omega_{1}$ and the negation of (1).

In particular, the strong form of Measuring* given by (3) in the above observation follows from ZF together with the Axiom of Determinacy.

We finish this digression into set theory without the Axiom of Choice by observing that any attempt to parametrize Measuring*, in the same vein as we did with Measuring, gives rise to principles vacuously equivalent to Measuring* itself, at least when the parametrization is done with the alephs ${ }^{2}$

Specifically, given an aleph $\kappa$, let us define Measuring* as the statement that for every sequence $\left\langle\mathcal{X}_{\alpha}: \alpha \in \omega_{1} \cap \operatorname{Lim}\right\rangle$, if each $\mathcal{X}_{\alpha}$ is a set of cardinality at most $\kappa$ consisting of subsets of $\alpha$, then there is a club $D \subseteq \omega_{1}$ such that for every limit point $\delta \in D$ of $D, D \cap \delta$ measures $X$ for all $X \in \mathcal{X}_{\delta}$. Then Measuring* ${ }_{\omega}^{*}$ is clearly equivalent to Measuring* under ZF together with the normality of $\mathcal{C}_{\omega_{1}}$ and the Axiom of Choice for countable families of subsets of $\omega_{1}$ (which of course follows from the Axiom of Choice for countable families of sets of reals, and therefore also from $Z F+A D)$. On the other hand, working in $Z F+\mathcal{C}_{\omega_{1}}$ is a normal filter on $\omega_{1}$, we have that Measuring $\omega_{1}^{*}$ follows vacuously from Measuring* simply because

\footnotetext{
${ }^{2}$ This was pointed out by Asaf Karagila.
} 
under Measuring* ${ }^{*}$ there is no sequence $\left\langle\mathcal{X}_{\alpha}: \alpha \in \omega_{1} \cap \operatorname{Lim}\right\rangle$ as in the definition of Measuring $\omega_{\omega_{1}}^{*}$ and such that $\left|\mathcal{X}_{\alpha}\right|=\omega_{1}$ for some $\alpha$; indeed, Measuring* implies, over this base theory, that $\mathcal{C}_{\omega_{1}}$ is an ultrafilter (Corollary 4.3 ), and if $\mathcal{C}_{\omega_{1}}$ is an ultrafilter then there is no $\omega_{1}$-sequence of distinct reals, whereas the existence of a family of size $\omega_{1}$ consisting of subsets of some fixed countable ordinal clearly implies that there is such a sequence.

We conclude the article with two natural questions.

Question 4.4. Is Measuring false?

Question 4.5. Are Measuring and Strong Measuring equivalent statements assuming Martin's Axiom?

\section{REFERENCES}

[1] U. Abraham. Proper forcing. In Handbook of set theory. Vols. 1, 2, 3, pages 333-394. Springer, Dordrecht, 2010.

[2] D. Asperó and M.A. Mota. Few new reals. Preprint.

[3] D. Asperó and M.A. Mota. Forcing consequences of PFA together with the continuum large. Trans. Amer. Math. Soc., 367:6103-6129, 2015.

[4] D. Asperó and M.A. Mota. A generalization of Martin's axiom. Israel J. Math., 210(1):193$231,2015$.

[5] D. Asperó and M.A. Mota. Separating club-guessing principles in the presence of fat forcing axioms. Ann. Pure Appl. Logic, 167(3):284-308, 2016.

[6] D. Asperó and M.A. Mota. Measuring club-sequences with the continuum large. J. Symbolic Logic, 82(3):1066-1079, 2017.

[7] J. Bagaria. Bounded proper forcing axioms as principles of generic absoluteness. Arch. Math. Logic, 39(6):393-401, 2000.

[8] T. Eisworth, D. Milovich, and J. Moore. Iterated forcing and the continuum hypothesis. In J. Cummings and E. Schimmerling, editors, Appalachian set theory 2006-2012, London Math. Soc. Lecture Notes series, pages 207-244. Cambridge Univ. Press, 2013.

[9] M. Goldstern and S. Shelah. The bounded proper forcing axiom. J. Symbolic Logic, 60(1):58$73,1995$.

[10] T. Miyamoto. $\omega_{1}$-Souslin trees under countable support iterations. Fund. Math., 142(3):257$261,1993$.

[11] T. Miyamoto and T. Yorioka. Some results in the extension with a coherent Suslin tree, part II (RIMS 2012). In RIMS Kokyuroku, number 1851, pages 49-61, 2013.

[12] J. T. Moore. Set mapping reflection. J. Math. Log., 5(1):87-97, 2005.

[13] S. Shelah. NNR revisited. Preprint.

[14] S. Shelah. Cardinal Arithmetic. Oxford Logic Guides, 29. Oxford Science Publications. The Clarendon Press, Oxford University Press, New York, 1994.

David Asperó, School of Mathematics, University of East Anglia, Norwich NR4 7TJ, $\mathrm{UK}$

E-mail address: d.aspero@uea.ac.uk

John Krueger, Department of Mathematics, University of North Texas, 1155 Union Circle \#311430, Denton, TX 76203

E-mail address: jkrueger@unt.edu 